\title{
Self-processing and the default mode network: interactions with the mirror neuron system
}

\author{
Istvan Molnar-Szakacs ${ }^{1,2}$ and Lucina Q. Uddin ${ }^{3,4}$ * \\ 1 Semel Institute for Neuroscience and Human Behavior, University of California, Los Angeles, CA, USA \\ 2 Tennenbaum Center for the Biology of Creativity, University of California, Los Angeles, CA, USA \\ ${ }^{3}$ Department of Psychiatry and Behavioral Sciences, Stanford University School of Medicine, Stanford, CA, USA \\ ${ }^{4}$ Department of Psychology, University of Miami, Coral Gables, FL, USA
}

\section{Edited by:}

Pengmin Qin, University of Ottawa Institute of Mental Health Research,

Canada

Reviewed by:

Sören Krach, Philipps-University

Marburg, Germany

Alessandra Ghinato Mainieri, RWTH

Aachen University, Germany

*Correspondence:

Lucina Q. Uddin, Department of

Psychiatry and Behavioral Sciences,

Stanford University School of

Medicine, 401 Quarry Road, Stanford,

CA 94305-5719, USA

e-mail: lucina@stanford.edu
Recent evidence for the fractionation of the default mode network (DMN) into functionally distinguishable subdivisions with unique patterns of connectivity calls for a reconceptualization of the relationship between this network and self-referential processing. Advances in resting-state functional connectivity analyses are beginning to reveal increasingly complex patterns of organization within the key nodes of the DMN - medial prefrontal cortex and posterior cingulate cortex - as well as between these nodes and other brain systems. Here we review recent examinations of the relationships between the DMN and various aspects of self-relevant and social-cognitive processing in light of emerging evidence for heterogeneity within this network. Drawing from a rapidly evolving social-cognitive neuroscience literature, we propose that embodied simulation and mentalizing are processes which allow us to gain insight into another's physical and mental state by providing privileged access to our own physical and mental states. Embodiment implies that the same neural systems are engaged for self- and other-understanding through a simulation mechanism, while mentalizing refers to the use of high-level conceptual information to make inferences about the mental states of self and others. These mechanisms work together to provide a coherent representation of the self and by extension, of others. Nodes of the DMN selectively interact with brain systems for embodiment and mentalizing, including the mirror neuron system, to produce appropriate mappings in the service of social-cognitive demands.

Keywords: functional connectivity, embodiment, mentalizing, autobiographical memory, medial prefrontal cortex, posterior cingulate cortex

\section{INTRODUCTION \\ DEFINING THE SELF AND BRAIN NETWORKS FOR SELF-RELATED PROCESSING}

The importance of self-knowledge has been asserted by philosophers, religious leaders, and thinkers cross-culturally. The Chinese philosopher Lao-Tzu claimed: "He who knows others is wise; He who knows himself is enlightened." The English cleric C. C. Colton wrote, "He that knows himself knows others, [...]," emphasizing the importance of self-knowledge for the sake of understanding others, as did Gandhi, who wrote, "He who knows himself, knows God and all others" (Gandhi, 1955). Throughout history, several examples exist of thinkers who have realized that representations of the self and others are intimately intertwined - that the self is a social stimulus. Current psychological theories suggest that the self may be considered a "special" stimulus, but also imply that it has similarities to other familiar and non-familiar stimuli that can be considered on a continuum of "familiarity" (e.g., kin recognition; Platek and Kemp, 2009) and "knowledge" (e.g., self-knowledge; Klein et al., 2002). For example, simulation theory proposes that in order to understand others we look inside ourselves to mentally simulate how we might act in given social situations (Gordon, 1986). Conversely, Gallotti and Frith (2013) have recently suggested that in order to understand ourselves, we pay close attention to the social behavior of others.

One major and useful distinction that has guided research on the neural representation of the self is that between the physical and psychological aspects of the self (Gillihan and Farah, 2005). Physical aspects of the self are typically examined in studies of selfface recognition, body recognition, agency, and perspective taking. Psychological aspects of the self tend to be operationalized with studies examining autobiographical memory and self-knowledge or self-referential processing (SRP) of personality traits. This conceptual distinction bears out in neuroimaging work, which suggests that physical or embodied self-related processes and psychological or evaluative self-related processes rely on distinct yet interacting large-scale brain networks (Lieberman, 2007; Uddin et al., 2007; Molnar-Szakacs and Arzy, 2009; Molnar-Szakacs and Uddin, 2012). For the purposes of the current review, the principal neural networks we will consider are the default mode network (DMN) and the human mirror neuron system (MNS).

The repeated observation that the medial prefrontal cortex (MPFC), posterior cingulate cortex (PCC), lateral parietal cortices, and medial temporal lobes paradoxically exhibit high levels of activity during resting baseline and decreases in activity 
during externally oriented cognitive tasks led to the initial characterization of these regions as belonging to a "default mode" of human brain function (Shulman et al., 1997; Gusnard and Raichle, 2001; Raichle et al., 2001; McKiernan et al., 2003; Fransson, 2006). This set of regions is more active when individuals rest than when they are engaged in goal-directed tasks. Importantly, these cortical regions tend to fluctuate in a coherent manner a phenomenon termed functional connectivity - which further supports the notion that they constitute a network of functionally related processing areas (Greicius et al., 2003; Fox et al., 2005; Golland et al., 2008). This network has also been referred to as the "task-negative network" (Fox et al., 2005), or the "cortical midline structures" (Northoff et al., 2006), and was originally proposed as a system for evaluating "information broadly arising in the external and internal milieu” (Raichle et al., 2001). The DMN has been posited to underlie a variety of general functions such as stimulus-independent (Mason et al., 2007) or task-unrelated thought (McKiernan et al., 2006), as well as socialcognitive or self-related processes, including episodic memory (Greicius and Menon, 2004), memory consolidation (Miall and Robertson, 2006), social processing (Iacoboni et al., 2004; Uddin et al., 2005), and various forms of self-related processing (Gusnard et al., 2001; Wicker et al., 2003b; Buckner and Carroll, 2007). More specifically, the DMN's involvement is observed most consistently during the psychological task of reflecting on one's own personality and characteristics (SRP), rather than during physical self-recognition (Qin and Northoff, 2011).

The MNS was first identified in non-human primates. Mirror neurons are active when an agent performs an action, and when it observes that same action being performed, in essence, creating an agent-independent connection between actor and observer (Rizzolatti and Sinigaglia, 2010). Based on the property of mirror neurons to internally simulate actions performed by others, it has been proposed that the MNS may provide the link between the physical representation of the self as related to the physical representation of others (Uddin et al., 2005, 2006, 2007). That is, when we see another's hand grasping an object, we activate the regions of our brain that control grasping; when we hear sounds associated with someone else's action, we activate the appropriate movement regions of our brain; and by extension, when we observe the emotional states of others, we can feel the same emotion in empathy (Carr et al., 2003; Gazzola et al., 2006; Molnar-Szakacs et al., 2006). These mirror-like processes are influenced by the observer's perspective and the goal of the action itself, which appears to be even more important than the way in which an action is performed (Gazzola et al., 2007). The brain regions involved in creating these interpersonal links include the MNS and its associated regions - the inferior frontal gyrus (IFG)/premotor cortex (PMC), the anterior insula $(\mathrm{AI})$, primary sensory and primary motor cortices, the inferior parietal lobule (IPL), and the superior temporal sulcus (STS).

The physical/psychological distinction, while perhaps simplistic, has facilitated the study of the neural networks underlying self-related processes. As the face is the most identifiable marker of the physical aspect of the self, it has been the subject of extensive study at the behavioral and neural levels. In particular, in our own work, we observed that the pattern of signal increases in the right
IFG and right IPL were related to the amount of self-face presented in morphed stimuli (morphed with the face of a familiar other). In other words, the greater amount of "self" present in the stimulus, the greater the activation in right fronto-parietal regions (Uddin et al., 2005). These regions overlap the human MNS, whose role is to map the actions of others onto one's own motor repertoire via a simulation mechanism (Rizzolatti et al., 1996). Similar findings have since been published (Sugiura et al., 2005; Platek et al., 2006; Uddin et al., 2006), supporting the role of the human MNS in physical self-recognition.

Psychological aspects of the self, such as those accessed through personality traits, likely evoke a representation of the self predominantly through linguistic aspects of the self-schema (Faust et al., 2004; Molnar-Szakacs et al., 2005b; Moran et al., 2006). Selfschemata are cognitive representations of the self that are derived from past social interactions and experiences and promote the elaboration of memories that may be used to guide future behavior (Markus, 1977). In one of the first neuroimaging studies on the subject, Kelley and colleagues used a trait adjective judgment task to compare processing of self-, other-, and case-referential adjectives. Results showed that the MPFC was selectively engaged in the self-related condition, while relevance judgments (i.e., "Does this adjective describe you/U.S. President George Bush?"), when compared to case judgments (i.e., "Is this adjective in lowercase letters?"), were accompanied by activation of the left IFG and the anterior cingulate cortex (ACC) (Kelley et al., 2002). This initial finding has since been replicated (Moran et al., 2009; Feyers et al., 2010), underscoring the role of MPFC in self-processing (Moran et al., 2013). Additionally, two recent meta-analyses have parcellated MPFC into ventral and dorsal aspects (Denny et al., 2012; Wagner et al., 2012), showing that ventral MPFC (VMPFC) responds more to self, and dorsal MPFC (DMPFC) responds more to others. Earlier work showed a similar dissociation along the lines of mentalizing about similar others (engaging VMPFC) and metalizing about dissimilar others (engaging DMPFC) (Mitchell et al., 2006).

Self-reference and self-relevance - whether by visual self-face recognition or through the enhanced memory for trait adjectives invoke autobiographical memory processes (Molnar-Szakacs and Arzy, 2009). Memory is vital to the survival of the self, as we use our memory for past events to predict the future and update action plans in a flexible, goal-oriented manner (for reviews, see Schacter et al., 2007, 2008). Recently, neuroimaging studies have started to investigate the neural networks subserving self-projection in time (Addis et al., 2007; Buckner and Carroll, 2007; Szpunar et al., 2007; Arzy et al., 2008). Arzy and colleagues used a paradigm that involved participants making mental self-projections to both past and future events, and found an effect of self, whereby participants responded significantly faster to self-relevant (personal) events than to non-self-relevant (world) events. Self-location in time was shown to recruit a distributed neural network - including anterior temporal, occipito-temporal, and temporo-parietal regions - that partly overlaps the DMN (Arzy et al., 2008). These brain regions were also recruited in studies of visuo-spatial perspective taking and spatial self-location (Vogeley and Fink, 2003; Blanke et al., 2005; Arzy et al., 2006). In one of the first descriptions of the DMN, Raichle et al. (2001) proposed a domain-general 
role for the PCC in providing complex visual representations to consciousness.

Taking into consideration the many facets of self-relevant processing such as self-face recognition, personality trait judgments, and autobiographical memory, it is not surprising that these processes recruit a vast network of brain regions. These include the human MNS for physical aspects of self-relevant processing, as well as the MPFC node of the DMN during SRP and the PCC/precuneus node of the DMN for self-location in time and space. In order to bridge the gaps between these neural and psychological levels of analysis, we need to correlate cognitive and affective experiences of self with the underlying neural processes supporting them. Inspired by current and historical psychological theories (Gordon, 1986; Gallotti and Frith, 2013) and extending upon our previous work (Molnar-Szakacs et al., 2005b; Uddin et al., 2005, 2006; Molnar-Szakacs and Uddin, 2012), we propose that many of the same neural systems are engaged for self- and other-understanding. Thus, having privileged access to our own physical and mental states allows us to gain insight into others' physical and mental states through the processes of embodiment and mentalizing. These cognitive processes are supported at the neural level by two large-scale, interacting networks - the MNS and the DMN, respectively. A more in-depth understanding of the functionally relevant nodes of each network, and the interactions between them, will help us advance toward a more complete theory of self-representation. By bringing together recent work on the fractionation of these complex networks, we aim to contribute to a more complete understanding of the self.

\section{NEURAL PROCESSES GIVING RISE TO THE SELF}

Preston and de Waal (2002) formalized a theory of emotionalmotor resonance in the Perception-Action Model, which holds that perception of a behavior performed by another automatically activates one's own representations for the behavior, and output from this shared representation automatically proceeds to motor areas of the brain where responses are prepared and executed. Emotional-motor resonance may also be called emotional empathy or embodied simulation - processes related to the same bottom-up, automatic, and evolutionarily early mechanism. Embodied simulation implies transforming perceived actions and emotions into our own inner representations of those actions and emotions. This process, supported by interactions between the MNS and the limbic system, is fast, automatic, and precognitive, and is thought to support our ability to empathize emotionally ("I feel what you feel") (Preston and de Waal, 2002). Current evolutionary evidence suggests that embodied simulation is a phylogenetically early system for empathy, and that there is also a more advanced cognitive perspective-taking (or theory of mind, ToM/mentalizing) system mediating empathic responses in humans (de Waal, 2008).

Higher-level cognitive empathy requires that we actively think about, or reflect on others' actions and emotional states, including perspective taking or ToM/mentalizing (de Waal, 2008). Mentalizing refers to the process of understanding another person's perspective, and appears to depend upon higher cognitive functions such as cognitive flexibility (Decety and Jackson, 2004). Singer (2006) has proposed that mentalizing allows us to understand mental states such as intentions, goals, and beliefs, while embodied simulation allows us to share the feelings of others. Low-level embodied processes and higher-level mentalizing processes integrate their signals such that stimuli are "mapped" onto internal representations and combined with information from memory to plan future behavior, select a response, and act. Neuroimaging studies have implicated distinct neural networks subserving embodiment and mentalizing processes (Shamay-Tsoory et al., 2004; Singer, 2006; Vollm et al., 2006; Hooker et al., 2008). Mentalizing processes appear to be centered on the MPFC node of the DMN, while embodied simulation processes are implemented by the MNS - limbic system network (Preston and de Waal, 2002; Gallese, 2005; Iacoboni and Dapretto, 2006; Iacoboni, 2009).

As previously discussed, the human MNS supports a simulation-based, motor resonance mechanism, whereby we understand the actions and emotions of others by "embodying" them ourselves. It has been suggested that mirror neurons are a kind of "neural wi-fi" that monitors what is happening in others. This system tracks others' emotions, what movements they're making, and what they intend, and activates in our brains precisely the same areas that are active in theirs. This puts us on the same wavelength and it does so "automatically, instantaneously and unconsciously" (Goleman, 2006). Neuroimaging studies have provided evidence in support of this notion, showing common neural signatures while experiencing disgust (Wicker et al., 2003a), touch (Keysers et al., 2004), or pain (Singer et al., 2004; Jackson et al., 2006) in oneself, and when perceiving the same feelings in others. Between-brain analyses have also provided evidence for neural resonance between individuals during social interactions (Schippers et al., 2010).

In thinking about the self and others, mentalizing representations (Barsalou, 1999, 2008) and embodied representations (Goldman and de Vignemont, 2009) serve as the foundations for making inferences about our own mind as well as others' minds. Recent work has suggested that higher-level inference-based mentalizing processes are grounded in their interactions with lowerlevel embodied simulation-based processes (Barsalou, 1999, 2008; Goldman, 2006; Keysers and Gazzola, 2007; Goldman and de Vignemont, 2009). This predicts that brain regions involved in high-level inference-based mentalizing are integrating their signals with lower-level simulation-based systems (Keysers and Gazzola, 2007; Uddin et al., 2007), implying DMN-MNS interactions during self-relevant processing (Sandrone, 2013). In a recent study, Schippers and Keysers have shown using Granger causal analyses that rather than simply being a feed-forward system in which visual representations are transformed into motor programs through a temporal $\rightarrow$ parietal $\rightarrow$ premotor flow of information, the MNS acts as a dynamic feedback control system, and that during gestural communication there is information flow within the system from premotor to parietal and temporal cortices (Schippers and Keysers, 2011). Their findings lend strong support to the notion of dynamic interactions between the MNS and the DMN.

Here we expand on recent theories linking embodiment and mentalizing systems (Keysers and Gazzola, 2007; Uddin et al., 2007; Molnar-Szakacs and Arzy, 2009; Paulus et al., 2013; Sandrone, 2013), and propose that the MNS and the DMN are functionally connected and dynamically interact during social-cognitive 
processing. Simulation-based representations serve to scaffold conceptual representations that allow us to understand the self in its social context. By virtue of their differential patterns of connectivity, subdivisions of the DMN can interact with the appropriate brain systems, including the MNS, in the service of self-related and social-cognitive demands. In light of recent work fractionating the DMN (Uddin et al., 2009; Andrews-Hanna et al., 2010), we will discuss some examples of how these low- and high-level mechanisms critical for representing the self are subserved by dissociable subdivisions of this network. In addition, we will highlight brain regions that may serve as key hubs mediating interactions between the DMN and MNS.

\section{DIFFERENT ASPECTS OF SELF-RELATED PROCESSING SELF-RELATED PROCESSING IN THE PHYSICAL DOMAIN}

One of the most important ways to identify one's own person is to recognize one's face and distinguish it from other persons' faces. Among the first to study the neural correlates of self-recognition in neurotypical adults, Keenan and colleagues provided behavioral (Keenan et al., 2000) and neural (Keenan et al., 2001) evidence for a right hemisphere bias in self-face processing. Subsequent functional Magnetic Resonance Imaging (fMRI) studies of self-face recognition described activations in lateral prefrontal cortex and parietal cortices during self-face recognition (Kircher et al., 2001; Platek et al., 2004, 2006; Sugiura et al., 2005). A recent review has highlighted the common finding of right frontal and parietal activations accompanying self-face viewing, especially when compared to other familiar faces (Devue and Bredart, 2011). Furthermore, a meta-analysis of studies of self-face recognition found that in addition to right fronto-parietal regions which overlap the human MNS, the right precuneus is a region that is also associated with this task (Platek et al., 2008). In our own work (Uddin et al., 2005), we provide clear evidence for a right hemisphere network including the IFG, IPL, superior parietal lobule, and inferior occipital gyrus activated by recognition of the self-face. The pattern of signal increases we observed in these areas as the stimuli contain more "self" suggest that these areas comprise a unique system extending beyond mere recognition of faces and play a particular role in self-face recognition. Perception of the self-face appears to involve a simulation-like mechanism that recruits right hemisphere MNS matching the face stimulus to an internal representation of the self. We proposed earlier that mirror areas may be more active for stimuli containing more "self" because their role is to establish communication between individuals via a simulation mechanism that maps actions of others onto one's own motor repertoire, thereby making others "like me" (Meltzoff and Brooks, 2001). Thus, when one sees one's own image, these mirror areas are more strongly activated because of the ease with which one can map oneself onto one's own motor system (Uddin et al., 2005). Interestingly, we also observed similar brain activation patterns distinguishing the self-voice from other voices, suggesting that the right hemisphere MNS may contribute to multimodal abstract self-representation (Kaplan et al., 2008).

Our results also demonstrated decreased activity within the DMN (precuneus, MPFC, and posterior superior temporal gyrus) only during processing of "self" stimuli (Uddin et al., 2005). This pattern of results led us to propose that the "familiar other" stimuli triggered social representations, and thus the task-related deactivation was compensated during viewing of the "other" by an increase in activity due to social processing. Thus, the overall result is lack of deactivation for "other," not a true activation. It is possible that during viewing of the "familiar other," with whom the subjects have a positive social relationship, the subjects automatically activate social representations to a greater extent than when viewing the "self." In summary, the generalized signal decrease in these DMN areas due to the task demands is offset in the "other" condition by triggering social-cognitive processing, which previously has been shown to engage these regions (Iacoboni et al., 2004). Thus, recognition of familiar others seems to also recruit midline structures that have previously been implicated in social processing (Saxe, 2006). Taken together, these results emphasize the importance of dynamic interactions between the MNS and the DMN during the processing of self-relevant information. The MNS appears to play an important role in physical self-recognition, while the DMN participates in situating the self in its social context relative to familiar others.

\section{SELF-REFERENTIAL PROCESSING IN THE VERBAL DOMAIN}

The self-reference effect (Symons and Johnson, 1997) is a unique encoding phenomenon, whereby memory for previously presented trait adjectives (e.g., happy) is better if they had been processed with reference to the self (e.g., "does happy describe you?") than if they had been processed only for their general meaning (e.g., "does happy mean the same as optimistic?"). In other words, as traits are incorporated into the self-schema, subsequent memory for these trait words is increased (Rogers et al., 1977). Several studies have used the self-reference effect to investigate SRP in the verbal domain. Using statements delivered through the auditory domain, Johnson and colleagues compared judgments about one's own abilities, traits, and attitudes (such as "I can be trusted") to a semantic judgment task. The self-referential condition was associated with activation in the MPFC and the PCC relative to the control condition (Johnson et al., 2002). Using a slightly different paradigm, Kjaer and colleagues asked participants to mentally induce thoughts reflecting on one's own personality traits and physical appearance. Once again, self-referential conditions induced activation in midline DMN regions including the MPFC and precuneus when compared to the non-self-referential conditions (Kjaer et al., 2002). They also observed increased functional connectivity between frontal and parietal midline regions during self-referential conditions. As evidenced by these studies, SRP in the verbal domain appears to recruit midline components of the DMN.

To tease apart the role of different subdivisions of the DMN in verbal SRP, Lou and colleagues used a combined PET-TMS approach. In the PET study, they used visually presented personality trait adjectives that were either related to the self, to the participants' best friend, or to the Danish Queen (Lou et al., 2004). Retrieval of self-related adjectives induced activation in the DMPFC, the PCC/precuneus, the right and left IPL, the left ventrolateral prefrontal cortex, and the middle temporal cortex including the hippocampus. As in previous studies, analysis of functional connectivity revealed significant interaction between anterior (DMPFC) and posterior (PCC, precuneus) midline regions 
of the DMN. Transcranial magnetic stimulation over the medial parietal region caused a decrease in the efficiency of retrieval of previous judgments of the mental self as compared to retrieval of judgments of others, confirming that this region may be a nodal structure in self-representation, mediating interactions between the DMN and other lateral cortical regions (Lou et al., 2004).

\section{SELF-REFERENTIAL PROCESSING IN THE MEMORY DOMAIN}

Self-referential processing in memory depends on the individual's life history and involves the recollection of past experiences, as the retrieved episodic information is unique to an individual and is tied to a specific personal context (Ingvar, 1985; Craik et al., 1999). Episodic memory retrieval (EMR), on the other hand, also includes the retrieval of events that are characterized by low selfrelevance. Behaviorally, the link between SRP and EMR is reflected in the so-called self-reference effect of memory, as discussed above (Rogers et al., 1977; Symons and Johnson, 1997). Further support for this link comes from neuroimaging investigations. EMR studies report activations in brain regions that are also identified by SRP tasks, including the MPFC, as well as the medial and lateral parietal cortex (Donaldson et al., 2001) (for reviews, see Cavanna and Trimble, 2006; Legrand and Ruby, 2009). Because these brain areas also show high neural activity during resting states, both SRP and EMR have been considered possible functions of the DMN (Buckner et al., 2008).

In a study designed to explore the similarity and dissociability of SRP and EMR, Sajonz and colleagues found that self-referential stimuli specifically activate the PCC/anterior precuneus, the MPFC, and an inferior division of the IPL. In contrast, EMR success specifically involves the posterior precuneus, the anterior prefrontal cortex, and a superior division of the IPL extending into the intraparietal sulcus and the superior parietal lobule. Overlapping activations can be found in intermediate zones in the precuneus and the IPL but not in the prefrontal cortex (Sajonz et al., 2010). These findings clearly demonstrate that distinct subdivisions of the DMN are recruited during SRP as compared with more general EMR. This is of particular interest in light of earlier studies associating the MPFC with autobiographical memory retrieval (Gilboa, 2004; Svoboda et al., 2006), retrieval of self-referential episodes (Zysset et al., 2002), retrieval of selfgenerated versus externally presented words (Vinogradov et al., 2008), and the self-reference effect of memory (Macrae et al., 2004). These processes have in common that they involve selfreferential and memory components at the same time. The data of Sajonz and colleagues seem to suggest that the self-referential component particularly contributes to activations of the medial prefrontal node of the DMN observed in these studies.

A functional connectivity analysis performed on the data suggests a functional segregation within the PCC/precuneus for SRP and EMR, respectively. Activity in the SRP-related seed in the PCC/anterior precuneus correlated with the MPFC, dorsal ACC, fusiform gyrus, and superior parietal lobule during SRP. In contrast, activity in the EMR-related seed in the posterior precuneus was associated with the responsiveness in a distinct region in the dorsal anterior paracingulate cortex during EMR (Sajonz et al., 2010). Taken together, these findings shed light on the parcellation of nodes within the DMN, and suggest that there is a functional segregation within the precuneus during SRP and EMR. Activity in anterior precuneus appears to be associated with SRP, a more self-directed process, whereas activity in posterior precuneus is associated with EMR, a more social and outward-directed process. This anterior/posterior functional parcellation within the precuneus mirrors the dorsal/ventral subdivision of the MPFC, as discussed above.

\section{NEURAL NETWORKS, FUNCTIONAL CONNECTIVITY, AND THE SELF FINDINGS FROM RESTING-STATE fMRI}

The past several years have witnessed a resurgence in the use of fMRI to study not only regional activation patterns in response to specific stimuli, but also functional connectivity between-brain regions both during task performance and during resting states. This focus on brain connectivity has emerged as a natural consequence of recent advances in methods for acquiring and analyzing resting-state fMRI data, as well as efforts such as the Human Connectome project (http://www. humanconnectomeproject.org/). Functional connectivity measured from fMRI data is defined as "temporal correlations between remote neurophysiological events" (Friston, 1994), and is typically quantified by conducting correlation analyses between regional timeseries (Cole et al., 2010). Since the initial demonstration that coherent low-frequency fluctuations in blood-oxygen-leveldependent (BOLD) signal index functionally significant brain systems (Biswal et al., 1995), the use of resting-state fMRI to characterize brain functional organization has sky-rocketed. This approach has been used to understand how the DMN might be further divided into functional subsystems.

It is often difficult to ascertain the functional roles of brain regions from their selective activation during processing of specific stimuli or associated with specific cognitive demands. Restingstate connectivity approaches, unconfounded by ceiling and floor effects in task performance, can provide complementary information regarding the functional roles of brain regions. It has been known since the initial study by Greicius et al. (2003) that brain areas comprising the DMN (PCC, MPFC, lateral parietal cortices), show coherent low-frequency fluctuations. Several recent studies examining the resting-state or intrinsic functional connectivity of the DMN have provided evidence for considerable heterogeneity between distinct nodes of the network. For example, the PCC has been shown to have stronger negative correlations with anterior cingulate and insular cortices, whereas the MPFC shows stronger negative correlations with posterior parietal cortices (Uddin et al., 2009). Several previous studies have demonstrated default mode suppression during goal-oriented task performance, with failure to suppress default mode activity being linked to decreased activity in task-relevant regions and attentional lapses, or decrements in performance (Weissman et al., 2006). Heterogeneity of DMN nodes in terms of their functional connectivity suggests that different avenues may exist for communicating with other brain systems critical for self-related processing.

While the MPFC and PCC are considered core "hubs" of the DMN, some have suggested that the network can be fractionated into subcomponents. Recently, Salomon et al. (2013) have proposed that the inferior and posterior parietal aspects of the DMN 
can be further subdivided such that some show greater involvement in self-referential judgments than others. Andrews-Hannah and colleagues found that one subsystem including DMPFC, temporo-parietal junction (TPJ), lateral temporal cortex, and temporal pole, is more engaged when individuals make self-referential judgments about their present situation or mental states, whereas a different subsystem comprised of VMPFC, medial temporal lobes, IPL, and retrosplenial cortex is more active during episodic judgments about the personal future (Andrews-Hanna et al., 2010). Others have subdivided the PCC into ventral and dorsal subdivisions. Leech et al. (2011) found that as difficulty increases during an N-back task, ventral PCC shows reduced integration within the DMN, whereas dorsal PCC shows increased integration with the DMN as well as attention networks. Taken together, these studies suggest that the concept of the DMN as a homogenous network should be refined and updated to account for heterogeneous patterns of activation and connectivity observed within the regions comprising it. This reconceptualization of the DMN as consisting of multiple interacting subsystems has clear implications for theories of the network's role in self-related cognition. In particular, the identification of possible "nodes of association" creating functional links enabling communication between the DMN and MNS are now beginning to be revealed. It has recently been demonstrated that certain brain regions constitute a "rich club" of organization in that they are highly connected hubs that are connected to other highly connected hubs (van den Heuvel and Sporns, 2011). We propose that such highly connected brain regions, including the $\mathrm{PCC} /$ precuneus and AI, may play a role in orchestrating dynamic interactions between the DMN and MNS.

\section{FUNCTIONS AND FUNCTIONAL CONNECTIVITY OF DMN NODES}

Although the precise functional properties of the DMN are not yet established, a growing number of studies implicate this network in various aspects of self-related processing. For example, the DMN is implicated during self-related evaluations (Northoff et al., 2006; Buckner and Carroll, 2007) voluntary actions (Goldberg et al., 2008), episodic memory (Spreng et al., 2009; Sestieri et al., 2011), and planning. Previous studies have revealed functional subdivisions within the DMN (Uddin et al., 2009; Andrews-Hanna et al., 2010; Sestieri et al., 2011) using either data driven parcellation methods (e.g., ICA, graph-analysis), or using specific tasks such as EMR. Within-region functional subdivisions in the DMN are also starting to be described as related to various neural processes including SRP and EMR (Andrews-Hanna et al., 2010; Sajonz et al., 2010; Kim, 2012) and cognitive control (Leech et al., 2011). In the following sections, we will describe some relevant studies that used a connectivity approach to explore DMN function and connectivity with the MNS and other brain regions during self-relevant processing.

Due to the overlap between brain regions involved in selfprocessing and regions that constitute the DMN (D'Argembeau et al., 2005; Schneider et al., 2008), some speak of a so-called "default self," arguing that the self may be more or less identical with the resting-state activity observed in DMN regions (Gusnard et al., 2001; Wicker et al., 2003b; Beer, 2007). A recent meta-analysis of 87 self-related studies has lent further support to this idea (Qin and Northoff, 2011). In their meta-analysis, Qin and Northoff asked a two-part question - is neural activity in the DMN selfspecific, and is self-specific activity related to resting-state activity? The specificity of the self (e.g., hearing one's own name, seeing one's own face) in the DMN was tested and compared across familiar (using stimuli from personally known people) and other (strangers and widely known figures) conditions. A large MPFC regions was recruited for the self condition when compared to the familiarity and other conditions. Concerning other midline regions, there was either regional overlap of activations between the self and familiarity conditions in the MPFC, or between the familiarity and other condition in the PCC (Qin and Northoff, 2011). This finding is in accordance with previous studies finding both self-specific and non-specific regions within the DMN during self-relevant processing (Gusnard et al., 2001; D'Argembeau et al., 2005; Schneider et al., 2008).

An interesting finding to emerge from the meta-analysis by Qin and Northoff (2011) was the recruitment of the right IFG, as well as the left AI during self-specific conditions. The role of the IFG as one of the anchors of the MNS and its role in self-relevant processing are well established (Molnar-Szakacs et al., 2005a; Uddin et al., 2005). As we have previously discussed, the right IFG seems to be responsive to self-face stimuli as well as one's own voice (Uddin et al., 2005; Kaplan et al., 2008). The insula has also been associated with self-specific stimuli in recent studies (Enzi et al., 2009; Modinos et al., 2009), and forms an integral part of the neural network important for emotional empathy, embodiment, and simulation (Carr et al., 2003; Singer et al., 2004). As the insula is heavily involved in interoceptive stimulus processing (Craig, 2003), one may suggest that the co-activation between insula and the DMN may be crucial in constituting the self and assigning self-specificity to stimuli. It has recently been demonstrated that the right AI plays a causal role in switching between the DMN and executive control networks (Sridharan et al., 2008). It has been suggested that the AI serves to detect events that are salient to the individual and mobilize neural resources in the service of appropriate behavioral responses (Menon and Uddin, 2010). That self-related stimuli should invoke activation of the insula is not surprising in light of these findings. Pre-reflective representations of visceral states of the self, for instance, seem linked to activations in the posterior and/or middle insula. By contrast, midline structures become active when subjects are asked to introspect, reflect, and report these states (e.g., heartbeat) (Critchley and Harrison, 2013). The AI seems crucial in linking the more posterior insula with these midline structures. Thus, interactions between the DMN and the MNS through the functional connectivity of midline structures and the AI could mediate the ability to represent one's bodily states to enable conscious reflection on those states (Keysers and Gazzola, 2007).

\section{INTERACTIONS BETWEEN THE DMN AND MNS}

The integration of function between the DMN and the MNS have been the focus of several recent proposals on the neural bases of self-related cognition (Keysers and Gazzola, 2007; Uddin et al., 2007; Molnar-Szakacs and Arzy, 2009; Molnar-Szakacs and Uddin, 2012; Paulus et al., 2013; Sandrone, 2013). The results of Qin and Northoff (2011) also lend support to the notion that the self emerges from the interaction of these two neural networks. Their 
meta-analysis showed recruitment of DMN regions, including the MPFC and PCC, as well as MNS regions, including the IFG and $\mathrm{AI}$, both during self-relevant processing.

Lombardo et al. (2010) used a functional connectivity approach to investigate the nature of the interaction between high-level mentalizing systems and embodied simulation-based representations during mentalizing and physical judgments about the self and others. The areas of overlap of activation between self and other consisted of the MPFC, PCC, and bilateral TPJ as well as the left anterior temporal lobe along the middle temporal gyrus, left primary sensorimotor cortex, and cerebellum. With a factorial design, they were able to test the interaction effect of whether mentalizing or physical representations recruit distinct functional circuits for the self or other. Similar patterns of functional connectivity between self and other conditions suggested that mentalizing representations are distributed across similar neural systems with respect to self and other. Conjunction analyses revealed a self-other distinction within the neural circuitry for mentalizing whereby the MPFC was biased for SRP, and the PCC and the TPJ were biased for other-referential processing, as has previously been shown (Ruby and Decety, 2001; Saxe et al., 2006; Pfeifer et al., 2007). As opposed to the previous within-region functional subdivisions we have discussed for the dorsal/ventral MPFC or the anterior/posterior precuneus, self-other distinction in this study mapped onto fronto-parietal DMN regions. Taken together, the results of these studies show that in addition to broad cross-regional functional specializations, region-specific functional specializations exist within nodes of the DMN.

A particularly interesting result of the study was that several MNS regions, including IFG/PMC, primary somatosensory cortex, and the AI were sensitive to processing of both self and other. The role of somatosensory cortex in low-level shared representations of touch (Keysers et al., 2004; Blakemore et al., 2005), self-experienced pain (Singer et al., 2004), and action-perception mirroring (Gazzola et al., 2006; Nanetti et al., 2009) is well established. Thus, the observation that primary somatosensory cortex is also recruited for mentalizing about self and other suggests that low-level embodied simulative representations computed by this region are also important for the processes underlying higher-level inference-based mentalizing when compared with reflecting on physical characteristics (Lombardo et al., 2010). In fact, connectivity analyses revealed that these two systems were specifically linked during mentalizing more than during physical judgments, and this pattern of connectivity was apparent for both self and other conditions. Taken together, these results provide strong evidence of the integration of function between the DMN and the MNS. The authors conclude that "the tight link between high-level inference-based mentalizing systems and lowlevel embodied/simulation-based systems suggests that these two neural systems for social cognition are integrated in a task-specific manner for mentalizing about both self and other" (Lombardo et al., 2010).

The studies reviewed here suggest that interactions between the DMN and MNS during self-relevant processing may occur through several associated brain regions. Figure 1 depicts some of the possible neuroanatomical loci and functional connections underlying such interactions.

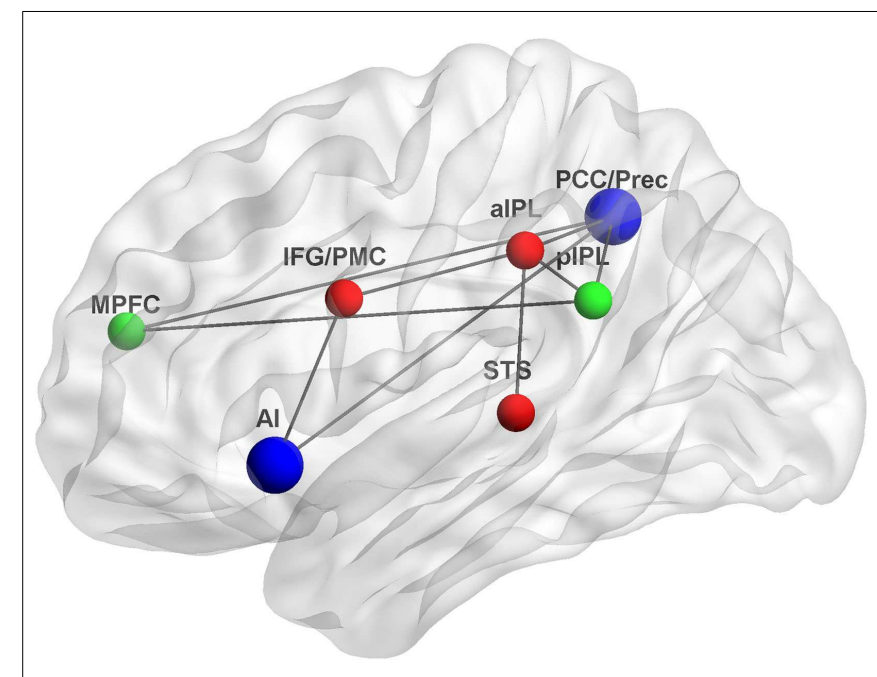

FIGURE 1 | Functional connections underlying interactions between the DMN and MNS. The DMN, a system for psychological self-relevant processing and mentalizing, and the MNS, a system for physical self-recognition and embodied simulation, may interact through densely connected "hubs" such as the Al and PCC/Prec. Green, DMN nodes; red, MNS nodes; blue, interaction nodes; MPFC, medial prefrontal cortex, pIPL, posterior inferior parietal lobule; PCC/Prec, posterior cingulate cortex/precuneus; IFG/PMC, inferior frontal gyrus/premotor cortex; aIPL, anterior inferior parietal lobule; STS, superior temporal sulcus; Al, anterior insula; Gray lines indicate possible functional connections based on (lacoboni et al., 2001; Lou et al., 2004; lacoboni and Dapretto, 2006; Sridharan et al., 2008; Schippers and Keysers, 2011). Figure was created using BrainNet Viewer (http://www.nitrc.org/projects/bnv/).

\section{CONCLUSION}

Historically, scholars have pitted high-level inference-based mentalizing accounts and low-level embodied simulation-based accounts as opposites of each other (Gopnik and Wellman, 1992; Gordon, 1992). However, recent theories related to different aspects of self-representation have been focused on the possible integration of function between the DMN and the MNS (Keysers and Gazzola, 2007; Uddin et al., 2007; Molnar-Szakacs and Arzy, 2009; Molnar-Szakacs and Uddin, 2012; Paulus et al., 2013; Sandrone, 2013). Furthermore, interpretations of disturbances in self-relevant processing often invoke explanations that are based either in deficits of the DMN, the human MNS, or both. For example, theories of how we understand other minds have implicated both the DMN (Spreng and Grady, 2009) and the MNS (Gallese and Goldman, 1998); theories about moral cognition have been linked to both the DMN (Harrison et al., 2008) and the MNS (Molnar-Szakacs, 2011); and both the DMN and the MNS have been implicated in theories of physical self-representation (Uddin et al., 2007; Molnar-Szakacs and Arzy, 2009; Molnar-Szakacs and Uddin, 2012). In the realm of psychiatric or neurological disorders, both the DMN (Cherkassky et al., 2006; Uddin, 2011) and the MNS (Iacoboni and Dapretto, 2006; Molnar-Szakacs et al., 2009; Enticott et al., 2012) have been implicated in autism spectrum disorders and aberrant DMN connectivity and MNS dysfunction have been observed in schizophrenia (Garrity et al., 2007; Mehta et al., 2012). Taken together, this evidence from both the 
healthy and the atypical brain suggests that the human MNS and the DMN are functionally connected and are together profoundly implicated in social cognition that forms the basis of understanding the self. In the context of situations requiring understanding of others' mental and physical states, such interactions facilitate the self-other mappings at the core of both embodiment and mentalizing processes.

Findings of functional specialization within the DMN are beginning to shed light on the ability of the network to support self-related processes as seemingly unrelated as autobiographical memory and verbal SRP. The findings reviewed here argue against viewing the DMN as a unitary system, and are compatible with the notion that the network consists of distinct, functionally specialized subsystems. It is becoming increasingly clear that great attention to anatomy can reveal subtle differences in circuitry of neighboring cortical regions of the DMN (Margulies et al., 2009). For example, we have seen that broad cross-regional functional specializations exist across regions of the DMN, such that the frontal MPFC node is more involved in self-related processing and the posterior PCC node is more involved in other-related processing. Additionally, region-specific functional specializations exist within nodes of the DMN, such that the VMPFC responds more

\section{REFERENCES}

Addis, D. R., Wong, A. T., and Schacter, D. L. (2007). Remembering the past and imagining the future: common and distinct neural substrates during event construction and elaboration. Neuropsychologia 45, 1363-1377. doi:10.1016/ j.neuropsychologia.2006.10.016

Andrews-Hanna, J. R., Reidler, J. S., Sepulcre, J., Poulin, R., and Buckner, R. L. (2010). Functional-anatomic fractionation of the brain's default network. Neuron 65, 550-562. doi: 10.1016/j.neuron.2010.02.005

Arzy, S., Molnar-Szakacs, I., and Blanke, O. (2008). Self in time: imagined self-location influences neural activity related to mental time travel. J. Neurosci. 28, 6502-6507. doi:10. 1523/JNEUROSCI.5712-07.2008

Arzy, S., Thut, G., Mohr, C., Michel, C. M., and Blanke, O. (2006). Neural basis of embodiment: distinct contributions of temporoparietal junction and extrastriate body area. J. Neurosci. 26, 8074-8081. doi:10. 1523/JNEUROSCI.0745-06.2006

Barsalou, L. W. (1999). Perceptual symbol systems. Behav. Brain Sci. 22, 577-609; discussion 610-560. doi: 10.1017/S0140525X99002149

Barsalou, L. W. (2008). Grounded cognition. Annu. Rev. Psychol. 59, 617-645. doi:10.1146/annurev. psych.59.103006.093639

Beer, J. S. (2007). The default self: feeling good or being right? Trends Cogn. Sci. (Regul. Ed.) 11, 187-189. doi:10. 1016/j.tics.2007.02.004
Biswal, B., Yetkin, F. Z., Haughton, V. M., and Hyde, J. S. (1995). Functional connectivity in the motor cortex of resting human brain using echo-planar MRI. Magn. Reson. Med. 34, 537-541. doi:10.1002/ mrm.1910340409

Blakemore, S. J., Bristow, D., Bird, G., Frith, C., and Ward, J. (2005). Somatosensory activations during the observation of touch and a case of vision-touch synaesthesia. Brain 128(Pt 7), 1571-1583. doi:10.1093/ brain/awh500

Blanke, O., Mohr, C., Michel, C. M., Pascual-Leone, A., Brugger, P., Seeck, M., et al. (2005). Linking out-ofbody experience and self processing to mental own-body imagery at the temporoparietal junction. $J$. Neurosci. 25, 550-557. doi:10.1523/ JNEUROSCI.2612-04.2005

Buckner, R. L., Andrews-Hanna, J. R., and Schacter, D. L. (2008). The brain's default network: anatomy, function, and relevance to disease. Ann. N. Y. Acad. Sci. 1124, 1-38. doi:10.1196/annals.1440.011 Self-projection and the brain. Trends Cogn. Sci. (Regul. Ed.) 11, 49-57. doi:10.1016/j.tics.2006.11.004

Carr, L., Iacoboni, M., Dubeau, M. C., Mazziotta, J. C., and Lenzi, G. L. (2003). Neural mechanisms of empathy in humans: a relay from neural systems for imitation to limbic areas. Proc. Natl. Acad. Sci. U.S.A. 100, 5497-5502. doi:10.1073/pnas. 0935845100
Buckner, R. L., and Carroll, D. C. (2007).

to self and the DMPFC responds more to others. Furthermore, emerging findings from the functional connectivity literature can greatly inform theories of DMN involvement in self-related cognition. In particular, they highlight possible avenues for interactions between the DMN and MNS, and indicate how brain networks for mentalizing and embodiment might communicate. Indeed, the studies discussed above suggest that the DMN and MNS may interact at certain "rich-club" nodes, including the $\mathrm{AI}$ and the PCC. Through this interaction, embodied simulation-based representations serve to scaffold mentalizing-based representations. These representations allow the brain to construct a dynamic self, continuous through time, and able to plan for the future. A more in-depth understanding of the functionally relevant nodes of each network, and the interactions between them, will help us advance toward a more complete theory of self-representation in the brain.

\section{ACKNOWLEDGMENTS}

This work was supported by a National Institute of Mental Health Career Development Award (K01MH092288) to Lucina Q. Uddin. The content is solely the responsibility of the authors and does not necessarily represent the official views of the NIMH or the NIH.

Cavanna, A. E., and Trimble, M. R. (2006). The precuneus: a review of its functional anatomy and behavioural correlates. Brain 129(Pt 3), 564-583. doi:10.1093/ brain/awl004

Cherkassky, V. L., Kana, R. K., Keller, T. A., and Just, M. A. (2006). Functional connectivity in a baseline restingstate network in autism. Neuroreport 17, 1687-1690. doi:10.1097/01.wnr. $0000239956.45448 .4 \mathrm{c}$

Cole, D. M., Smith, S. M., and Beckmann, C. F. (2010). Advances and pitfalls in the analysis and interpretation of resting-state FMRI data. Front. Syst. Neurosci. 4:8. doi:10. 3389/fnsys.2010.00008

Craig, A. D. (2003). Interoception: the sense of the physiological condition of the body. Curr. Opin. Neurobiol. 13, 500-505. doi:10.1016/ S0959-4388(03)00090-4

Craik, F. I. M., Moroz, T. M., Moscovitch, M., Stuss, D. T., Winocur, G., Tulving, E., et al. (1999). In search of the self: a positron emission tomography study. Psychol. Sci. 10, 26-34. doi:10.1111/1467-9280.00102

Critchley, H. D., and Harrison, N. A. (2013). Visceral influences on brain and behavior. Neuron 77, 624-638. doi:10.1016/j.neuron. 2013.02.008

D'Argembeau, A., Collette, F., van der Linden, M., Laureys, S., Del Fiore, G., Degueldre, C., et al. (2005) Self-referential reflective activity and its relationship with rest: a PET study. Neuroimage 25,
616-624. doi:10.1016/j.neuroimage. 2004.11.048

de Waal, F. B. M. (2008). Putting the altruism back into altruism: the evolution of empathy. Annu. Rev. Psychol. 59, 279-300. doi:10.1146/ annurev.psych.59.103006.093625

Decety, J., and Jackson, P. L. (2004). The functional architecture of human empathy. Behav. Cogn. Neurosci. Rev. 3, 71-100. doi:10.1177/1534582304267187

Denny, B. T., Kober, H., Wager, T. D., and Ochsner, K. N. (2012). A metaanalysis of functional neuroimaging studies of self- and other judgments reveals a spatial gradient for mentalizing in medial prefrontal cortex. J. Cogn. Neurosci. 24, 1742-1752. doi:10.1162/jocn_a_00233

Devue, C., and Bredart, S. (2011). The neural correlates of visual selfrecognition. Conscious. Cogn. 20, 40-51. doi:10.1016/j.concog.2010. 09.007

Donaldson, D. I., Petersen, S. E., and Buckner, R. L. (2001). Dissociating memory retrieval processes using fMRI: evidence that priming does not support recognition memory. Neuron 31, 1047-1059. doi:10.1016/ S0896-6273(01)00429-9

Enticott, P. G., Kennedy, H. A., Rinehart, N. J., Tonge, B. J., Bradshaw, J. L., Taffe, J. R., et al. (2012). Mirror neuron activity associated with social impairments but not age in autism spectrum disorder. Biol. Psychiatry 71, 427-433. doi:10.1016/j. biopsych.2011.09.001 
Enzi, B., de Greck, M., Prosch, U., Tempelmann, C., and Northoff, G. (2009). Is our self nothing but reward? Neuronal overlap and distinction between reward and personal relevance and its relation to human personality. PLoS ONE 4:e8429. doi:10.1371/journal.pone. 0008429

Faust, M., Kravetz, S., and Nativ-Safrai, O. (2004). The representation of aspects of the self in the two cerebral hemispheres. Pers. Individ. Dif. 37, 607-619. doi:10.1016/j.paid. 2003.09.030

Feyers, D., Collette, F., D’Argembeau, A., Majerus, S., and Salmon, E. (2010). Neural networks involved in self-judgement in young and elderly adults. Neuroimage 53, 341-347. doi:10.1016/j.neuroimage. 2010.05.071

Fox, M. D., Snyder, A. Z., Vincent, J. L., Corbetta, M., van Essen, D. C., and Raichle, M. E. (2005). The human brain is intrinsically organized into dynamic, anticorrelated functional networks. Proc. Natl. Acad. Sci. U.S.A. 102, 9673-9678. doi:10.1073/ pnas.0504136102

Fransson, P. (2006). How default is the default mode of brain function? Further evidence from intrinsic BOLD signal fluctuations. Neuropsychologia 44, 2836-2845. doi:10.1016/j. neuropsychologia.2006.06.017

Friston, K. (1994). Functional and effective connectivity in neuroimaging: a synthesis. Hum. Brain Mapp. 2, 56-78. doi:10.1002/hbm.460020107

Gallese, V. (2005). “"Being like me”: selfother identity, mirror neurons, and empathy," in Perspectives on Imitation: Mechanisms of Imitation and Imitation in Animals, Vol. 2, eds S. Hurley and N. Chater (Cambridge, MA: MIT Press), 101-118.

Gallese, V., and Goldman, A. (1998). Mirror neurons and the simulation theory of mind-reading. Trends Cogn. Sci. (Regul. Ed.) 2, 493-501. doi:10.1016/S13646613(98)01262-5

Gallotti, M., and Frith, C. D. (2013). Social cognition in the we-mode. Trends Cogn. Sci. (Regul. Ed.) 17, 160-165. doi:10.1016/j.tics.2013.02. 002

Gandhi, M. K. (1955). My Religion. Ahmedabad: Navajivan Publishing House.

Garrity, A. G., Pearlson, G. D., McKiernan, K., Lloyd, D., Kiehl, K. A., and Calhoun, V. D. (2007). Aberrant "default mode" functional connectivity in schizophrenia. Am. J. Psychiatry 164, 450-457. doi:10.1176/appi. ajp. 164.3 .450
Gazzola, V., Aziz-Zadeh, L., and Keysers, C. (2006). Empathy and the somatotopic auditory mirror system in humans. Curr. Biol. 16, 1824-1829. doi:10.1016/j.cub.2006.07.072

Gazzola, V., Rizzolatti, G., Wicker, B., and Keysers, C. (2007). The anthropomorphic brain: the mirror neuron system responds to human and robotic actions. Neuroimage 35, 1674-1684. doi:10.1016/ j.neuroimage.2007.02.003

Gilboa, A. (2004). Autobiographical and episodic memory - one and the same? Evidence from prefrontal activation in neuroimaging studies. Neuropsychologia 42, 1336-1349. doi:10.1016/j.neuropsychologia. 2004.02.014

Gillihan, S. J., and Farah, M. J. (2005). Is self special? A critical review of evidence from experimental psychology and cognitive neuroscience. Psychol. Bull. 131, 76-97. doi:10.1037/ 0033-2909.131.1.76

Goldberg, I., Ullman, S., and Malach, R. (2008). Neuronal correlates of "free will" are associated with regional specialization in the human intrinsic/default network. Conscious. Cogn. 17, 587-601. doi:10.1016/j.concog.2007.10.003

Goldman, A., and de Vignemont, F (2009). Is social cognition embodied? Trends Cogn. Sci. (Regul. Ed.) 13, 154-159. doi:10.1016/j.tics.2009.01. 007

Goldman, A. I. (2006). Simulating Minds: The Philosophy, Psychology, and Neuroscience of Mindreading. New York: Oxford University Press.

Goleman, D. (2006). Social Intelligence: The New Science of Human Relationships. New York: Random House.

Golland, Y., Golland, P., Bentin, S., and Malach, R. (2008). Data-driven clustering reveals a fundamental subdivision of the human cortex into two global systems. Neuropsychologia 46, 540-553. doi:10.1016/j. neuropsychologia.2007.10.003

Gopnik, A., and Wellman, H. M. (1992) Why the child's theory of mind really is a theory. Mind Lang. 7, 145-171. doi:10.1111/j.1468-0017. 1992.tb00202.x

Gordon, R. M. (1986). Folk psychology as simulation. Mind Lang. 1, 158-171. doi:10.1111/j.1468-0017. 1986.tb00324.x

Gordon, R. M. (1992). The simulation theory: objections and misconceptions. Mind Lang. 7, 11-34. doi:10. 1111/j.1468-0017.1992.tb00195.x

Greicius, M. D., Krasnow, B., Reiss, A. L., and Menon, V. (2003). Functional connectivity in the resting brain: a network analysis of the default mode hypothesis. Proc. Natl. Acad. Sci. U.S.A. 100, 253-258. doi:10.1073/ pnas. 0135058100

Greicius, M. D., and Menon, V. (2004). Default-mode activity during a passive sensory task: uncoupled from deactivation but impacting activation. $J$ Cogn. Neurosci. 16, 1484-1492. doi:10.1162/0898929042568532

Gusnard, D. A., Akbudak, E., Shulman, G. L., and Raichle, M. E. (2001) Medial prefrontal cortex and selfreferential mental activity: relation to a default mode of brain function. Proc. Natl. Acad. Sci. U.S.A 98, 4259-4264. doi:10.1073/pnas. 071043098

Gusnard, D. A., and Raichle, M. E. (2001). Searching for a baseline: functional imaging and the resting human brain. Nat. Rev. Neurosci. 2 685-694. doi:10.1038/35094500

Harrison, B. J., Pujol, J., Lopez-Sola, M., Hernandez-Ribas, R., Deus, J., Ortiz, H., et al. (2008). Consistency and functional specialization in the default mode brain network. Proc. Natl. Acad. Sci. U.S.A. 105, 9781-9786. doi:10.1073/pnas. 0711791105

Hooker, C. I., Verosky, S. C., Germine, L. T., Knight, R. T., and D'Esposito, M. (2008). Mentalizing about emotion and its relationship to empathy. Soc. Cogn. Affect. Neurosci. 3, 204-217. doi:10.1093/scan/nsn019

Iacoboni, M. (2009). Imitation, empathy, and mirror neurons. Annu. Rev. Psychol. 60, 653-670. doi:10.1146/ annurev.psych.60.110707.163604

Iacoboni, M., and Dapretto, M. (2006). The mirror neuron system and the consequences of its dysfunction. Nat. Rev. Neurosci. 7, 942-951. doi: 10.1038/nrn2024

Iacoboni, M., Koski, L. M., Brass, M. Bekkering, H., Woods, R. P., Dubeau, M. C., et al. (2001). Reafferent copies of imitated actions in the right superior temporal cortex. Proc. Natl. Acad. Sci. U.S.A. 98, 13995-13999. doi:10.1073/pnas.241474598

Iacoboni, M., Lieberman, M. D., Knowlton, B. J., Molnar-Szakacs, I., Moritz, M., Throop, C. J., et al. (2004). Watching social interactions produces dorsomedial prefrontal and medial parietal BOLD fMRI signal increases compared to a resting baseline. Neuroimage 21, 1167-1173. doi 10.1016/j.neuroimage.2003.11.013

Ingvar, D. H. (1985). "Memory of the future": an essay on the temporal organization of conscious awareness. Hum. Neurobiol. 4, 127-136.

Jackson, P. L., Brunet, E., Meltzoff, A. N., and Decety, J. (2006). Empathy examined through the neural mechanisms involved in imagining how I feel versus how you feel pain. Neuropsychologia 44, 752-761. doi:10.1016/j.neuropsychologia. 2005.07.015

Johnson, S. C., Baxter, L. C., Wilder, L. S., Pipe, J. G., Heiserman, J. E., and Prigatano, G. P. (2002). Neural correlates of self-reflection. Brain 125(Pt 8), 1808-1814. doi:10.1093/ brain/awf181

Kaplan, J. T., Aziz-Zadeh, L., Uddin, L. Q., and Iacoboni, M. (2008). The self across the senses: an fMRI study of self-face and self-voice recognition. Soc. Cogn. Affect. Neurosci. 3, 218-223. doi:10.1093/scan/ nsn014

Keenan, J. P., Freund, S., Hamilton, R. H., Ganis, G., and Pascual-Leone, A. (2000). Hand response differences in a self-face identification task. $\mathrm{Neu}$ ropsychologia 38, 1047-1053. doi:10. 1016/S0028-3932(99)00145- 1

Keenan, J. P., Nelson, A., O'Connor, M., and Pascual-Leone, A. (2001) Self-recognition and the right hemisphere. Nature 409, 305. doi:10. 1038/35053167

Kelley, W. M., Macrae, C. N., Wyland, C. L., Caglar, S., Inati, S., and Heatherton, T. F. (2002). Finding the self? An event-related fMRI study. J. Cogn. Neurosci. 14, 785-794. doi:10.1162/ 08989290260138672

Keysers, C., and Gazzola, V. (2007). Integrating simulation and theory of mind: from self to social cognition. Trends Cogn. Sci. (Regul. Ed.) 11, 194-196. doi:10.1016/j.tics.2007.02. 002

Keysers, C., Wicker, B., Gazzola, V., Anton, J. L., Fogassi, L., and Gallese, V. (2004). A touching sight: SII/PV activation during the observation and experience of touch. Neuron 42,335-346. doi:10.1016/S08966273(04)00156-4

Kim, H. (2012). A dual-subsystem model of the brain's default network: self-referential processing, memory retrieval processes, and autobiographical memory retrieval. $\mathrm{Neu}$ roimage 61, 966-977. doi:10.1016/j. neuroimage.2012.03.025

Kircher, T. T., Senior, C., Phillips, M. L., Rabe-Hesketh, S., Benson, P. J., Bullmore, E. T., et al. (2001). Recognizing one's own face. Cognition 78, B1-B15. doi:10.1016/S00100277(00)00104-9

Kjaer, T. W., Nowak, M., and Lou, H. C. (2002). Reflective self-awareness and conscious states: PET evidence for a common midline parietofrontal core. Neuroimage 17, 1080-1086. doi:10.1006/nimg.2002.1230 
Klein, S. B., Cosmides, L., Costabile, K. A., and Mei, L. (2002). Is there something special about the self? A neuropsychological case study. J. Res. Pers. 36, 490-506. doi:10.1016/ S0092-6566(02)00001-6

Leech, R., Kamourieh, S., Beckmann, C. F., and Sharp, D. J. (2011). Fractionating the default mode network: distinct contributions of the ventral and dorsal posterior cingulate cortex to cognitive control. J. Neurosci. 31, 3217-3224. doi:10.1523/ JNEUROSCI.5626-10.201

Legrand, D., and Ruby, P. (2009). What is self-specific? Theoretical investigation and critical review of neuroimaging results. Psychol. Rev. 116,252-282. doi:10.1037/a0014172

Lieberman, M. D. (2007). Social cognitive neuroscience: a review of core processes. Annu. Rev. Psychol. 58, 259-289. doi:10.1146/annurev. psych.58.110405.085654

Lombardo, M. V., Chakrabarti, B., Bullmore, E. T., Wheelwright, S. J., Sadek, S. A., Suckling, J., et al. (2010). Shared neural circuits for mentalizing about the self and others. $J$. Cogn. Neurosci. 22, 1623-1635. doi: 10.1162/jocn.2009.21287

Lou, H. C., Luber, B., Crupain, M., Keenan, J. P., Nowak, M., Kjaer, T. W., et al. (2004). Parietal cortex and representation of the mental self. Proc. Natl. Acad. Sci. U.S.A. 101, 6827-6832. doi:10.1073/pnas. 0400049101

Macrae, C. N., Moran, J. M., Heatherton, T. F., Banfield, J. F., and Kelley, W. M. (2004). Medial prefrontal activity predicts memory for self. Cereb. Cortex 14, 647-654. doi:10.1093/cercor/ bhh025

Margulies, D. S., Vincent, J. L., Kelly, C., Lohmann, G., Uddin, L. Q., Biswal, B. B., et al. (2009). Precuneus shares intrinsic functional architecture in humans and monkeys. Proc. Natl. Acad. Sci. U.S.A. 106, 20069-20074. doi:10.1073/pnas.0905314106

Markus, H. (1977). Self-schemata and processing information about the self. J. Pers. Soc. Psychol. 35, 63-78. doi:10.1037/0022-3514.35.2.63

Mason, M. F., Norton, M. I., van Horn, J. D., Wegner, D. M., Grafton, S. T., and Macrae, C. N. (2007). Wandering minds: the default network and stimulus-independent thought. Science 315, 393-395. doi:10.1126/ science. 1131295

McKiernan, K. A., D’Angelo, B. R., Kaufman, J. N., and Binder, J. R. (2006). Interrupting the "stream of consciousness": an fMRI investigation. Neuroimage 29, 1185-1191. doi:10. 1016/j.neuroimage.2005.09.030
McKiernan, K. A., Kaufman, J. N., Kucera-Thompson, J., and Binder, J. R. (2003). A parametric manipulation of factors affecting task-induced deactivation in functional neuroimaging. J. Cogn. Neurosci. 15, 394-408. doi:10.1162/089892903321593117

Mehta, U. M., Basavaraju, R., Thirthalli, J., and Gangadhar, B. N. (2012). Mirror neuron dysfunction-a neuromarker for social cognition deficits in drug naive schizophrenia. Schizophr. Res. 141, 281-283. doi:10.1016/ j.schres.2012.07.005

Meltzoff, A., and Brooks, R. (2001). " "Like me" as a building block for understanding other minds: bodily acts, attention and intention," in Intentions and Intentionality: Foundations of Social Cognition, eds B. Malle, L. Moses, and D. Baldwin (Cambridge: MIT Press), 171-191.

Menon, V., and Uddin, L. Q. (2010). Saliency, switching, attention and control: a network model of insula function. Brain Struct. Funct. 214, 655-667. doi:10.1007/s00429-0100262-0

Miall, R. C., and Robertson, E. M. (2006). Functional imaging: is the resting brain resting? Curr. Biol. 16, R998-R1000. doi:10.1016/j.cub. 2006.10.041

Mitchell, J. P., Macrae, C. N., and Banaji, M. R. (2006). Dissociable medial prefrontal contributions to judgments of similar and dissimilar others. Neuron 50, 655-663. doi:10. 1016/j.neuron.2006.03.040

Modinos, G., Ormel, J., and Aleman, A. (2009). Activation of anterior insula during self-reflection. PLoS ONE 4:e4618. doi:10.1371/journal. pone.0004618

Molnar-Szakacs, I. (2011). From actions to empathy and morality - a neural perspective. J. Econ. Behav. Organ. 77, 76-85. doi:10.1016/j.jebo.2010. 02.019

Molnar-Szakacs, I., and Arzy, S. (2009). Searching for an integrated selfrepresentation. Commun. Integr. Biol. 2, 365-367. doi:10.4161/cib.2. 4.8290

Molnar-Szakacs, I., Iacoboni, M., Koski, L., and Mazziotta, J. C. (2005a). Functional segregation within pars opercularis of the inferior frontal gyrus: evidence from fMRI studies of imitation and action observation. Cereb. Cortex 15, 986-994. doi:10.1093/cercor/ bhh199

Molnar-Szakacs, I., Uddin, L. Q., and Iacoboni, M. (2005b). Righthemisphere motor facilitation by self-descriptive personalitytrait words. Eur. J. Neurosci. 21, 2000-2006. doi:10.1111/j.14609568.2005.04019.x

Molnar-Szakacs, I., Kaplan, J., Greenfield, P. M., and Iacoboni, M. (2006). Observing complex action sequences: the role of the frontoparietal mirror neuron system. $\mathrm{Neu}$ roimage 33, 923-935. doi:10.1016/j. neuroimage.2006.07.035

Molnar-Szakacs, I., and Uddin, L. Q. (2012). "The emergent self: how distributed neural networks support self-representation," in Handbook of Neurosociology, Handbooks of Sociology and Social Research, eds D. D. Franks and J. H. Turner (New York: Springer), 167-182.

Molnar-Szakacs, I., Wang, M. J., Laugeson, E. A., Overy, K., Wu, W. L., and Piggot, J. (2009). Autism, emotion recognition and the mirror neuron system: the case of music. Mcgill J. Med. 12, 87 .

Moran, J. M., Heatherton, T. F., and Kelley, W. M. (2009). Modulation of cortical midline structures by implicit and explicit self-relevance evaluation. Soc. Neurosci. 4, 197-211. doi:10.1080/17470910802250519

Moran, J. M., Kelley, W. M., and Heatherton, T. F. (2013). What can the organization of the brain's default mode network tell us about self-knowledge? Front. Hum Neurosci. 7:391. doi:10.3389/fnhum. 2013.00391

Moran, J. M., Macrae, C. N., Heatherton, T. F., Wyland, C. L., and Kelley, W. M. (2006). Neuroanatomical evidence for distinct cognitive and affective components of self. J. Cogn. Neurosci. 18 1586-1594. doi:10.1162/jocn.2006. 18.9.1586

Nanetti, L., Cerliani, L., Gazzola, V. Renken, R., and Keysers, C. (2009). Group analyses of connectivitybased cortical parcellation using repeated k-means clustering. $\mathrm{Neu}$ roimage 47, 1666-1677. doi:10.1016/ j.neuroimage.2009.06.014

Northoff, G., Heinzel, A., de Greck, M., Bermpohl, F., Dobrowolny, H., and Panksepp, J. (2006). Selfreferential processing in our brain a meta-analysis of imaging studies on the self. Neuroimage 31 , 440-457. doi:10.1016/j.neuroimage. 2005.12.002

Paulus, F. M., Muller-Pinzler, L., Westermann, S., and Krach, S. (2013). On the distinction of empathic and vicarious emotions. Front. Hum. Neurosci. 7:196. doi:10.3389/fnhum. 2013.00196
Pfeifer, J. H., Lieberman, M. D., and Dapretto, M. (2007). "I know you are but what am I?!": neural bases of self- and social knowledge retrieval in children and adults. J. Cogn. Neurosci. 19, 1323-1337. doi:10.1162/ jocn.2007.19.8.1323

Platek, S. M., Keenan, J. P., Gallup, G. G. Jr., and Mohamed, F. B (2004). Where am I? The neurological correlates of self and other. Brain Res. Cogn. Brain Res. 19, 114-122. doi:10.1016/j.cogbrainres. 2003.11.014

Platek, S. M., and Kemp, S. M. (2009). Is family special to the brain? An event-related fMRI study of familiar, familial, and selfface recognition. Neuropsychologia 47, 849-858. doi:10.1016/j. neuropsychologia.2008.12.027

Platek, S. M., Loughead, J. W., Gur, R. C., Busch, S., Ruparel, K., Phend, N., et al. (2006). Neural substrates for functionally discriminating self-face from personally familiar faces. Hum. Brain Mapp. 27, 91-98. doi:10.1002/ hbm. 20168

Platek, S. M., Wathne, K., Tierney, N. G. and Thomson, J. W. (2008). Neural correlates of self-face recognition: an effect-location meta-analysis. Brain Res. 1232, 173-184. doi:10.1016/j. brainres.2008.07.010

Preston, S. D., and de Waal, F. B. (2002). Empathy: its ultimate and proximate bases. Behav. Brain Sci. 25, 1-20; discussion 20-71.

Qin, P., and Northoff, G. (2011). How is our self related to midline regions and the default-mode network? Neuroimage 57, 1221-1233. doi:10. 1016/j.neuroimage.2011.05.028

Raichle, M. E., MacLeod, A. M., Snyder, A. Z., Powers, W. J., Gusnard, D. A., and Shulman, G. L. (2001). A default mode of brain function. Proc. Natl. Acad. Sci. U.S.A. 98, 676-682. doi:10.1073/pnas.98.2.676

Rizzolatti, G., Fadiga, L., Gallese, V., and Fogassi, L. (1996). Premotor cortex and the recognition of motor actions. Brain Res. Cogn. Brain Res. 3, 131-141. doi:10.1016/09266410(95)00038-0

Rizzolatti, G., and Sinigaglia, C. (2010). The functional role of the parietofrontal mirror circuit: interpretations and misinterpretations. Nat. Rev. Neurosci. 11, 264-274. doi:10. 1038/nrn2805

Rogers, T. B., Kuiper, N. A., and Kirker, W. S. (1977). Self-reference and the encoding of personal information. J. Pers. Soc. Psychol. 35, 677-688. doi:10.1037/0022-3514.35.9.677

Ruby, P., and Decety, J. (2001). Effect of subjective perspective taking during 
simulation of action: a PET investigation of agency. Nat. Neurosci. 4, 546-550.

Sajonz, B., Kahnt, T., Margulies, D. S., Park, S. Q., Wittmann, A., Stoy, M., et al. (2010). Delineating self-referential processing from episodic memory retrieval: common and dissociable networks. Neuroimage 50, 1606-1617. doi:10.1016/j. neuroimage.2010.01.087

Salomon, R., Levy, D. R., and Malach, R. (2013). Deconstructing the default: cortical subdivision of the default mode/intrinsic system during selfrelated processing. Hum. Brain Mapp. doi:10.1002/hbm.22268

Sandrone, S. (2013). Self through the mirror (neurons) and default mode network: what neuroscientists found and what can still be found there. Front. Hum. Neurosci. 7:383. doi:10. 3389/fnhum.2013.00383

Saxe, R. (2006). Uniquely human social cognition. Curr. Opin. Neurobiol. 16, 235-239. doi:10.1016/j. conb.2006.03.001

Saxe, R., Moran, J. M., Scholz, J., and Gabrieli, J. (2006). Overlapping and non-overlapping brain regions for theory of mind and self reflection in individual subjects. Soc. Cogn. Affect. Neurosci. 1, 229-234. doi:10.1093/ scan/nsl034

Schacter, D. L., Addis, D. R., and Buckner, R. L. (2007). Remembering the past to imagine the future: the prospective brain. Nat. Rev. Neurosci. 8, 657-661. doi:10.1038/ nrn2213

Schacter, D. L., Addis, D. R., and Buckner, R. L. (2008). Episodic simulation of future events: concepts, data, and applications. Ann. N. Y. Acad. Sci. 1124,39-60. doi:10.1196/annals. 1440.001

Schippers, M. B., and Keysers, C. (2011). Mapping the flow of information within the putative mirror neuron system during gesture observation. Neuroimage 57, 37-44. doi:10.1016/ j.neuroimage.2011.02.018

Schippers, M. B., Roebroeck, A., Renken, R., Nanetti, L., and Keysers, C. (2010). Mapping the information flow from one brain to another during gestural communication. Proc. Natl. Acad. Sci. U.S.A. 107, 9388-9393. doi:10.1073/pnas. 1001791107

Schneider, F., Bermpohl, F., Heinzel, A., Rotte, M., Walter, M., Tempelmann, C., et al. (2008). The resting brain and our self: self-relatedness modulates resting state neural activity in cortical midline structures. Neuroscience 157, 120-131. doi:10. 1016/j.neuroscience.2008.08.014
Sestieri, C., Corbetta, M., Romani, G. L., and Shulman, G. L. (2011). Episodic memory retrieval, parietal cortex, and the default mode network: functional and topographic analyses. J. Neurosci. 31, 4407-4420. doi:10. 1523/JNEUROSCI.3335-10.2011

Shamay-Tsoory, S. G., Tomer, R., Goldsher, D., Berger, B. D., and AharonPeretz, J. (2004). Impairment in cognitive and affective empathy in patients with brain lesions: anatomical and cognitive correlates. J. Clin. Exp. Neuropsychol. 26, 1113-1127. doi:10.1080/ 13803390490515531

Shulman, G. L., Corbetta, M., Fiez, J. A., Buckner, R. L., Miezin, F. M., Raichle, M. E., et al. (1997). Searching for activations that generalize over tasks. Hum. Brain Mapp. 5, 317-322. doi: 10.1002/(SICI)1097-0193(1997)5:4

Singer, T. (2006). The neuronal basis and ontogeny of empathy and mind reading: review of literature and implications for future research. Neurosci. Biobehav. Rev. 30, 855-863. doi:10.1016/j.neubiorev.2006.06. 011

Singer, T., Seymour, B., O’Doherty, J., Kaube, H., Dolan, R. J., and Frith, C. D. (2004). Empathy for pain involves the affective but not sensory components of pain. Science 303, 1157-1162. doi:10.1126/ science. 1093535

Spreng, R. N., and Grady, C. L. (2009). Patterns of brain activity supporting autobiographical memory, prospection, and theory-of-mind and their relationship to the default mode network. J. Cogn. Neurosci. 22, 1112-1123. doi:10.1162/jocn.2009. 21282

Spreng, R. N., Mar, R. A., and Kim, A. S. (2009). The common neural basis of autobiographical memory, prospection, navigation, theory of mind, and the default mode: a quantitative meta-analysis. J. Cogn. Neurosci. 21, 489-510. doi:10.1162/jocn. 2008.21029

Sridharan, D., Levitin, D. J., and Menon, V. (2008). A critical role for the right fronto-insular cortex in switching between central-executive and default-mode networks. Proc. Natl. Acad. Sci. U.S.A. 105, 12569-12574. doi:10.1073/pnas.0800005105

Sugiura, M., Watanabe, J., Maeda, Y., Matsue, Y., Fukuda, H., and Kawashima, R. (2005). Cortical mechanisms of visual selfrecognition. Neuroimage 24, 143-149. doi:10.1016/j.neuroimage. 2004.07.063

Svoboda, E., McKinnon, M. C., and Levine, B. (2006). The functional neuroanatomy of autobiographical memory: a meta-analysis. Neuropsychologia 44, 2189-2208. doi:10.1016/j.neuropsychologia. 2006.05.023

Symons, C. S., and Johnson, B. T (1997). The self-reference effect in memory: a meta-analysis. Psychol. Bull. 121, 371-394. doi:10.1037/ 0033-2909.121.3.371

Szpunar, K. K., Watson, J. M. and McDermott, K. B. (2007) Neural substrates of envisioning the future. Proc. Natl. Acad. Sci. U.S.A. 104, 642-647. doi:10.1073/pnas.0610082104

Uddin, L. Q. (2011). Brain connectivity and the self: the case of cerebral disconnection. Conscious. Cogn. 20, 94-98. doi:10.1016/ j.concog.2010.09.009

Uddin, L. Q., Iacoboni, M., Lange, C. and Keenan, J. P. (2007). The self and social cognition: the role of cortical midline structures and mirror neurons. Trends Cogn. Sci. (Regul. Ed.) 11,153-157. doi:10.1016/j.tics.2007. 01.001

Uddin, L. Q., Kaplan, J. T., MolnarSzakacs, I., Zaidel, E., and Iacoboni, M. (2005). Self-face recognition activates a frontoparietal "mirror" network in the right hemisphere: an event-related fMRI study. $\mathrm{Neu}$ roimage 25, 926-935. doi:10.1016/j. neuroimage.2004.12.018

Uddin, L. Q., Kelly, A. M., Biswal, B. B., Castellanos, F. X., and Milham, M. P. (2009). Functional connectivity of default mode network components: correlation, anticorrelation and causality. Hum. Brain Mapp. 30 625-637. doi:10.1002/hbm.20531

Uddin, L. Q., Molnar-Szakacs, I., Zaidel, E., and Iacoboni, M. (2006). rTMS to the right inferior parietal lobule disrupts self-other discrimination. Soc. Cogn. Affect. Neurosci. 1, 65-71. doi:10.1093/scan/ns1003

van den Heuvel, M. P., and Sporns, O. (2011). Rich-club organization of the human connectome J. Neurosci. 31, 15775-15786. doi:10.1523/JNEUROSCI.3539- 11. 2011

Vinogradov, S., Luks, T. L., Schulman, B. J., and Simpson, G. V. (2008). Deficit in a neural correlate of reality monitoring in schizophrenia patients. Cereb. Cortex 18, 2532-2539. doi:10. 1093/cercor/bhn028

Vogeley, K., and Fink, G. R. (2003). Neural correlates of the first-personperspective. Trends Cogn. Sci. (Regul. Ed.) 7, 38-42. doi:10.1016/S13646613(02)00003-7

Vollm, B. A., Taylor, A. N., Richardson, P., Corcoran, R., Stirling, J.,
McKie, S., et al. (2006). Neuronal correlates of theory of mind and empathy: a functional magnetic resonance imaging study in a nonverbal task. Neuroimage 29, 90-98. doi:10.1016/j.neuroimage.2005.07. 022

Wagner, D. D., Haxby, J. V., and Heatherton, T. F. (2012). The representation of self and person knowledge in the medial prefrontal cortex. Wiley Interdiscip. Rev. Cogn. Sci. 3, 451-470. doi:10.1002/wcs.1183

Weissman, D. H., Roberts, K. C., Visscher, K. M., and Woldorff, M. G. (2006). The neural bases of momentary lapses in attention. Nat. Neurosci. 9, 971-978. doi:10.1038/ nn1727

Wicker, B., Keysers, C., Plailly, J., Royet, J. P., Gallese, V., and Rizzolatti, G. (2003a). Both of us disgusted in my insula: the common neural basis of seeing and feeling disgust. Neuron 40, 655-664. doi:10.1016/S08966273(03)00679-2

Wicker, B., Ruby, P., Royet, J. P., and Fonlupt, P. (2003b). A relation between rest and the self in the brain? Brain Res. Brain Res. Rev. 43, 224-230. doi:10.1016/j.brainresrev. 2003.08.003

Zysset, S., Huber, O., Ferstl, E., and von Cramon, D. Y. (2002). The anterior frontomedian cortex and evaluative judgment: an fMRI study. Neuroimage 15, 983-991. doi:10.1006/nimg. 2001.1008

Conflict of Interest Statement: The authors declare that the research was conducted in the absence of any commercial or financial relationships that could be construed as a potential conflict of interest.

Received: 24 May 2013; accepted: 26 August 2013; published online: 11 September 2013.

Citation: Molnar-Szakacs I and Uddin LQ (2013) Self-processing and the default mode network: interactions with the mirror neuron system. Front. Hum. Neurosci. 7:571. doi: 10.3389/fnhum.2013.00571 This article was submitted to the journal Frontiers in Human Neuroscience.

Copyright (C) 2013 Molnar-Szakacs and Uddin. This is an open-access article distributed under the terms of the Creative Commons Attribution License (CC BY). The use, distribution or reproduction in other forums is permitted, provided the original author(s) or licensor are credited and that the original publication in this journal is cited, in accordance with accepted academic practice. No use, distribution or reproduction is permitted which does not comply with these terms. 